\title{
Long-Term Underwater Hydrophobicity: Exploring Topographic and Chemical Requirements
}

\author{
Aránzazu Martínez-Gómez, Silvia López, Teresa García, Raquel de Francisco, Pilar Tiemblo,* \\ and Nuria García*๑ \\ Instituto de Ciencia y Tecnología de Polímeros, ICTP-CSIC, Juan de la Cierva 3, 28006 Madrid, Spain
}

Supporting Information

ABSTRACT: A family of hybrid organoinorganic silica-based particles with varied chemical natures and morphologies has been synthesized to test their ability to develop coatings with underwater hydrophobicity. The particles were characterized by elemental microanalysis, scanning electron microscopy, and dynamic light scattering to evaluate the organic content, observe the morphology, and estimate the aggregate size, respectively. These morphologies were transferred into surface topographies by spraycoating dispersions made from the particles onto glass supports, resulting in coatings with an

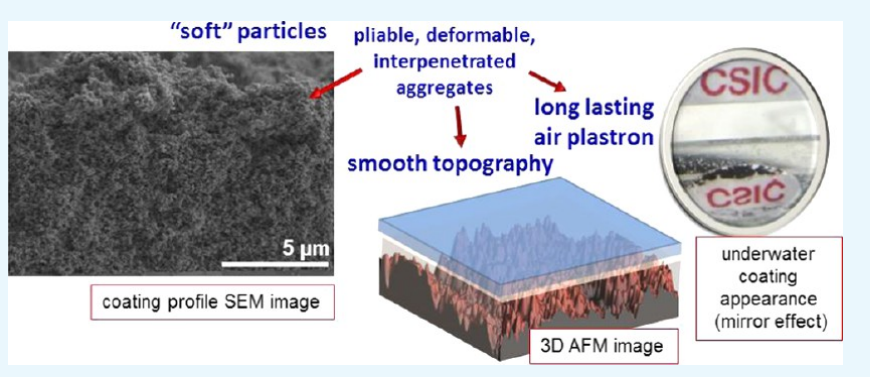
ample range of profiles and roughness but all of them being superhydrophobic. Atomic force microscopy and optical profilometry were used to map the coating surfaces and analyze the topography. Then, underwater hydrophobicity endurance was tested by immersion under a $2 \mathrm{~cm} 20{ }^{\circ} \mathrm{C}$ water column perpendicular to circular glass supports coated with the particles. The so-called mirror effect derived from the occurrence of the primary plastron (continuous air layer occluded between the surface and the water) was observed on the surface of all of the coatings tested. Apart from the dependency of plastrons on the water temperature and substrate shape, the plastron quality and lifetime is notably different depending on the particle morphology and thus on the coating topography. These experiments have demonstrated that the most persistent mirror effects, and therefore underwater superhydrophobicity, were produced on coatings that exhibited the smoothest topographies at the micrometric scale. In addition, these particle-only coatings can be made mechanically stable and robust by blending with a polymer matrix.

\section{INTRODUCTION}

Research on superhydrophobic surfaces is currently an attractive topic not only because of the many issues that remain to be understood but also because of their vast technological implications, such as water repellent, selfcleaning, antifouling, anticorrosive, drag reduced, or anti-icing surfaces among others. ${ }^{1,2}$ The term superhydrophobic accounts for a surface that presents an apparent (global) water contact angle above $150^{\circ}$ and a hysteresis (the difference between advancing and receding water contact angles, which gives an idea of the water adhesion to the surface) under $10^{\circ} .3$ These surfaces behave as totally water repellent due to the combination of two circumstances: low surface energy chemistry and a characteristic surface roughness. The development of such a surface is nowadays quite straightforward by using different approaches, for example, lithography, chemical etching, electrospinning, phase separation, crystallization, particle deposition, layer by layer, etc. ${ }^{4}$ However, what is more complex is to meet, together with the superhydrophobic character, some other desirable requirements, such as mechanical stability, transparency, or resistance to long water contact or immersion. The latter is probably the most intriguing factor because it is less well known and underexplored but is extremely useful in practice.
During the first half of the 20th century, research on the respiration of aquatic insects led to the discovery of gas plastrons stuck to the insects, that is, an air cushion trapped in between water and the insect's body that could be sustained, even for years, because of gas being extracted from aerated water. $^{5}$ It was not until 2006 that an attempt to produce a synthetic material able to mimic such a mechanism was published by Shirtcliffe et al., ${ }^{6}$ who presented a submerged methyltrimethoxysilane foam able to extract gases from water, as insects do. In 2007, a different strategy consisting of the galvanic deposition of a metal on a metallic substrate was employed by Bell and co-workers, ${ }^{7}$ who claimed to be the first to report the mirror-like reflection (mirror effect), which can be visualized on the submerged surface at a certain glancing angle because of the total internal reflection caused by the different refraction index of water and the trapped air in the plastron. Since 2009, when the first quantitative measurements of plastron reflectivity decay were published, ${ }^{8}$ the number of articles dealing with plastrons on synthetic materials has largely increased, both because of the novelty of the phenomenon (in synthetic materials) and because of its many technological

Received: November 3, 2017

Accepted: November 30, 2017

Published: December 13, 2017 
Table 1. Size, Polydispersity, and Organic Incorporation of the Silica Particles and Contact Angles of the Coatings Prepared from the Particle Suspensions

\begin{tabular}{|c|c|c|c|c|c|c|c|}
\hline \multirow[b]{2}{*}{ sample } & \multirow[b]{2}{*}{ synthetic method } & \multirow[b]{2}{*}{ modifier } & \multirow[b]{2}{*}{ OM\% } & \multicolumn{2}{|c|}{ ethanol suspension } & \multicolumn{2}{|c|}{ coating } \\
\hline & & & & $Z_{\text {ave }}(\mathrm{nm})$ & polydispersity index (Pdi) & $\theta_{\mathrm{w}}(\mathrm{deg})$ & $t_{\text {mirror }}(\mathrm{h})$ \\
\hline P7 & physisorption & PDMS-7 & 8.8 & $188 \pm 10$ & $0.15 \pm 0.01$ & $163 \pm 2$ & $340 \pm 12$ \\
\hline P662 & & PDMS-662 & 27.7 & $2532 \pm 300$ & $0.31 \pm 0.06$ & $167 \pm 4$ & $72 \pm 8$ \\
\hline PFS & & PTFPMS & 7.6 & $184 \pm 15$ & $0.18 \pm 0.02$ & $165 \pm 2$ & $96 \pm 8$ \\
\hline $\operatorname{Sm} 7$ & Stöber condensation & PDMS-7 & 9.9 & $730 \pm 20$ & $0.41 \pm 0.10$ & $164 \pm 2$ & $60 \pm 6$ \\
\hline $\operatorname{Sm} 57$ & & PDMS-57 & 11.1 & $700 \pm 64$ & $0.17 \pm 0.08$ & $162 \pm 1$ & $3 \pm 0.2$ \\
\hline $\mathrm{SmF}$ & & Fluorolink & 10.2 & $364 \pm 13$ & $0.28 \pm 0.08$ & $154 \pm 2$ & $48 \pm 8$ \\
\hline SmFS & & PTFPMS & 32.8 & $310 \pm 25$ & $0.51 \pm 0.12$ & $160 \pm 3$ & $4 \pm 0.2$ \\
\hline $\mathrm{Fm} 7$ & fluoride-catalyzed condensation & PDMS-7 & 25.3 & $270 \pm 18$ & $0.52 \pm 0.08$ & $157 \pm 2$ & $36 \pm 6$ \\
\hline $\mathrm{FmF}$ & & Fluorolink & 39.8 & $1442 \pm 225$ & $0.32 \pm 0.05$ & $155 \pm 2$ & $50 \pm 8$ \\
\hline FmFS & & PTFPMS & 35.0 & $256 \pm 12$ & $0.30 \pm 0.02$ & $160 \pm 3$ & $144 \pm 12$ \\
\hline SMTMS & self-condensation & MTMS & & $895 \pm 108$ & $0.16 \pm 0.04$ & $150 \pm 2$ & $1 \pm 0.2$ \\
\hline
\end{tabular}

implications, such as underwater breathing, antifouling or anticorrosive applications, drag reduction, nonsticking coatings, for example for packaging applications, etc.

Even though the principles for plastron development and regeneration over time had been studied almost a century before in insects, much of the work published since 2008 on the duration of plastrons underwater does not describe the experiments sufficiently, and except for the pressure of the water column, other basic experimental parameters (temperature or aeration of water) are frequently not mentioned. Bobji et al., ${ }^{9}$ who studied the possibility of synthetic materials maintaining plastrons for long times, described that the experiment is carried at $20{ }^{\circ} \mathrm{C}$ in deionized water that is left standing for a couple of days prior to its use. However, they did not mention the influence of temperature nor found differences in plastron duration when aerating the water. Poetes et al. ${ }^{10}$ were the first to actually visualize the air plastron on a Teflon surface as it decays by using confocal microscopy. They were able to show how the primary plastron is, as time goes by, transformed into many air caps that characterize a secondary plastron. They presented data on the duration of the plastron as a function of depth, but they did not mention the temperature of the experiments, although they did say that the plastron is very dependent on conditions like aeration of the water or flow.

Highlighting a difference between primary and secondary plastrons, as these authors do, is not banal because although all underwater applications rely on long-term hydrophobicity, not all of them rely on it in the same way, and although antifouling or anticorrosion can be very possibly achieved effectively with secondary plastrons, other applications, such as nonsticking coatings, drag reduction, or underwater breathing, require a significant and continuous layer of trapped air, i.e, a primary plastron. In this regard, plastron reversibility is also of great interest. In 2011, ${ }^{11}$ the first work (as far as we know) on the reversibility of the Cassie Wenzel transition appeared, in the context of the application of underwater superhydrophobicity to microfluidics. In this work, the effect of pressure (increase and decrease) on plastron formation is studied, and it is seen that a decrease in pressure can lead to the formation of a plastron after it is lost, what they believe is proof of a WenzelCassie transition. In recent years, more evidence on the dynamics of plastrons has been provided. ${ }^{12,13}$ However, it is still not clear whether there is real reversibility, as the dynamics of plastrons seem to evidence in these two last works for example, or not, as other authors suggest. ${ }^{14,15}$ Molecular simulations of density fluctuations indicate, according to some authors, that spontaneous dewetting and recovery of the plastron may occur. $^{16}$

Because plastron occurrence is a relatively new phenomenon in synthetic materials, experimental evidence of its characteristics is still very necessary. Recently, we have studied how the control of the aggregate size of surface-modified organosilica particles enables the production of transparent superhydrophobic coatings ${ }^{17,18}$ with similar surface chemistry, which can develop a large variety of topographical features. In this work, we evaluate their underwater hydrophobicity in shallow water experiments and their ability to produce and sustain welldeveloped plastrons. The aim is the study of the influence of experimental factors such as geometry of the substrate, or water temperature and water temperature variations in plastron duration, and the identification of the topographical features most relevant in this phenomenon, which will help to design and build a stable and long-lasting underwater superhydrophobic surface.

\section{RESULTS AND DISCUSSION}

The methodology applied proposes to synthesize two types of organosilica, soft and hard particles. ${ }^{19}$ Soft organosilicas consist of flexible and deformable agglomerates that can interpenetrate each other, and they can be built by condensation reactions using a silica precursor, such as tetraethyl orthosilicate (TEOS), or by surface modification of commercial silica nanoparticles. On the contrary, hard organosilicas organize themselves into impenetrable and undeformable particles or aggregates, and they can be synthesized by condensation reactions with TEOS and/or organic comonomers. Hard organosilicas are discrete entities whereas soft organosilicas organize themselves into entities (aggregates and agglomerates) ${ }^{20,21}$ that are pliable and easily deformed by external stresses, including swelling when in contact with certain solvents.

Both types of particles allow control over the topography of the coatings obtained after the deposition of the particles onto the substrate ${ }^{22}$ and also the preparation of the ample variety of topographies required for the purpose of this work.

Table 1 collects the characterization of the modified silica particles and coatings employed in this work: organic incorporation (organic mass (OM\%)) determined by elemental analysis, size and polydispersity measured in an ethanol dispersion by dynamic light scattering (DLS), contact angles of the coatings, and underwater mirror duration. All of these coatings have in common that they show $\theta_{\mathrm{w}}>150^{\circ}$, that water droplets effortlessly roll off ( $\Delta \theta$ values are not reported for the 
sake of simplicity but they are under $10^{\circ}$ in all cases), and that they are able to sustain a primary plastron, even if it is a shortlived one (see Table 1). For the rest, the coatings have been prepared to be as diverse as possible to evaluate the structural features associated with the development and evolution of plastrons. Four different synthetic methods and four different organic modifiers have been employed, leading to organosilica particles and aggregates whose structure, organic incorporation, size, and polydispersity are strongly varied.

The synthetic method and the modifier obviously determine the OM\%, which varies from less than 8 wt \% to almost $40 \mathrm{wt}$ $\%$. Physisorption is the less effective method to incorporate organic mass for the same modifier, compare samples P7, Sm7, and Fm7. On the contrary, fluoride-catalyzed condensation results in the highest OM\%. Increasing the molar mass of the modifier mostly results in an increase in OM\%, see for example, P662 compared to P7 or Sm57 compared to Sm7. It also has to be taken into account that in samples obtained by physisorption, the organic mass is attached to the outer surface, because they are synthesized from silica particles by surface physisorption of the modifier. In the condensation methods, TEOS is supposed to act as a cross-linker preventing the growth of long siloxane chains, ${ }^{23}$ although "core-shell" and other different structures have also been described in this kind of co-condensation reaction. ${ }^{24,25}$ The organosilicas prepared in these ways will range from the "hard" to the "soft", a characteristic which will be of importance when preparing the coatings with them.

The ${ }^{29} \mathrm{Si}$ cross-polarization/magic-angle spinning (CP/MAS) NMR spectra of some representative samples are collected in Figure 1. The spectra exhibit two characteristic resonance

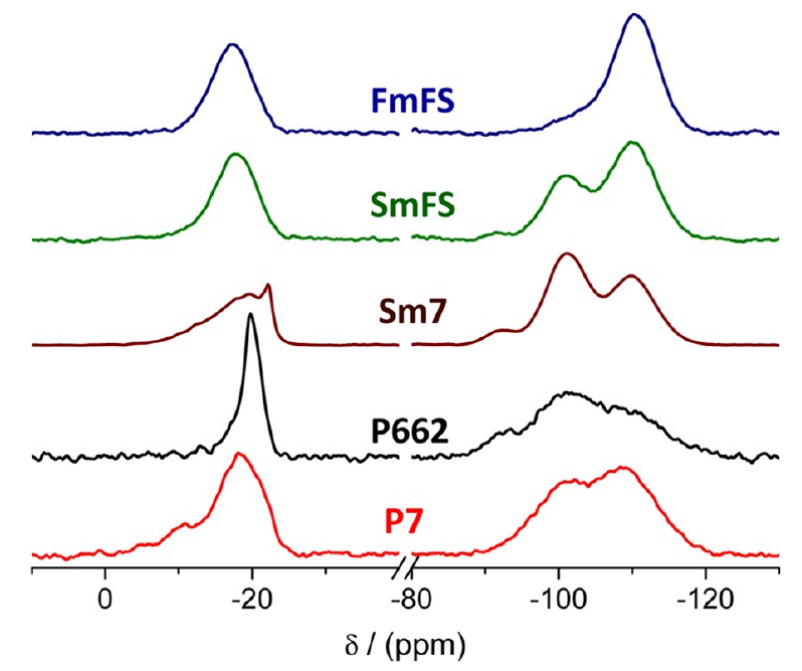

Figure 1. ${ }^{29} \mathrm{Si} \mathrm{CP} / \mathrm{MAS}$ NMR spectra of some of the synthesized particles.

regions, ${ }^{26,27}$ the inorganic region, $\mathrm{Q}$ centered at around -105 ppm, assigned to silanols, $\mathrm{Si}(\mathrm{O})_{4-x}(\mathrm{OH})_{x}$, siloxane groups, and $\mathrm{Si}(\mathrm{O})_{4}$, and the organic region, $\mathrm{D}$, associated with $\mathrm{Si}(\mathrm{C})_{2}(\mathrm{O})_{2}$ siloxane groups at around $-20 \mathrm{ppm}$. The structure of both regions depends on the synthetic method and the modifier employed. Uncondensed silanols (signals $\mathrm{Q}^{3}$ and $\mathrm{Q}^{2}$ at -100 and $-90 \mathrm{ppm}$, respectively) are more visible in the samples prepared by physisorption and Stöber condensation, whereas fluoride-catalyzed reactions seem to result in significant TEOS condensation ( $\mathrm{Q}$ region appears practically as a single band centered at the position of the $\mathrm{Q}^{4}$ species, $\mathrm{Si}(\mathrm{OSi})_{4}$, around $-110 \mathrm{ppm})$. Regarding the $\mathrm{D}$ region, it is known that free liquid-like polydimethylsiloxane (PDMS) units appear at -22 $\mathrm{ppm},{ }^{28}$ so the closer the signal is to $-22 \mathrm{ppm}$, the higher the mobility of the chains. As expected, P662, modified with the longest PDMS chain, exhibits a D region centered the closest to $-22 \mathrm{ppm}$, indicating that the modifier chains represent relative free motion. On the contrary, the particles prepared with poly(trifluoropropylmethylsiloxane) (PTFPMS), either by fluoride or Stöber condensation, are those whose D region appeared broader and centered at low field, showing a more restricted environment of the siloxane units. In any case, the modifier was successfully incorporated into the particle in all of the samples, giving rise to a hybrid structure. The only nonhybrid particle is SMTMS (NMR spectrum shows only an organic region, $\mathrm{T}$, centered at $-60 \mathrm{ppm}){ }^{29}$ produced by the self-condensation of a trifunctional organosilane (methyltrimethoxysilane (MTMS)).

The large number and variety of samples, all of them superhydrophobic, allows comparison of the different surface features and searching for those that can best provide long-term underwater hydrophobicity. With this objective in mind, we carried out experiments consisting of immersing the coatings under a $2 \mathrm{~cm}$ water column and studying the formation and duration of an air plastron. Pictures showing the aspect of the coatings a few minutes after immersion are shown in Figure 2.

All of the prepared coatings produce well-developed plastrons under the immersion conditions described in the Experimental Section, visualized as "mirrors" appearing on the sample surface at given glancing angles. Differences in the uniformity of the mirrors throughout the sample surfaces are seen depending on the particles, and some of them are even able to very easily produce large macroscopic primary plastrons (see, for example, P7, Sm7, and SmF in Figure 2).

However, the most notable difference among the samples is the mirror lifetimes, which are listed in Table 1. Figure 3 presents three pictures showing how the quality, continuity, and homogeneity of the mirror in the SmF coating is lost with time. After losing the large macroscopic primary plastron covering the whole sample (Figure 3a), smaller macroscopic plastrons remain that are still able to produce clear image reflections (Figure $3 b$ ). Reflexivity progressively decreases as the macroscopic plastrons are lost, and the surface acquires a silvery aspect that is characteristic of the development of many small submacroscopic air caps that give rise to the silver shine (Figure $3 c)$. When the surface appears that way, the $t_{\text {mirror }}$ is considered to end in this work, even if the surface may still be reflecting light to a certain extent.

Before getting into details, it is worth mentioning that experiments estimating mirror lifetimes have to be well defined, so that comparison between different samples makes sense (or comparison between results from different laboratories). Although efforts to quantify the reflection from the plastron have been done, ${ }^{8}$ experimental factors that very strongly influence its duration are often overlooked. Actually, the main dependency of the plastron and the mirror effect duration is definitively the height of the water column (the pressure endured by the air layer), which is well reported in the literature. ${ }^{9,10}$ We decided to use a relatively small water column $(2 \mathrm{~cm})$ so that we could study an ample set of coatings, including those where plastrons were formed but lasted for very short times. Otherwise, if a higher water column had been used, the mirror could have disappeared even during the filling of the 

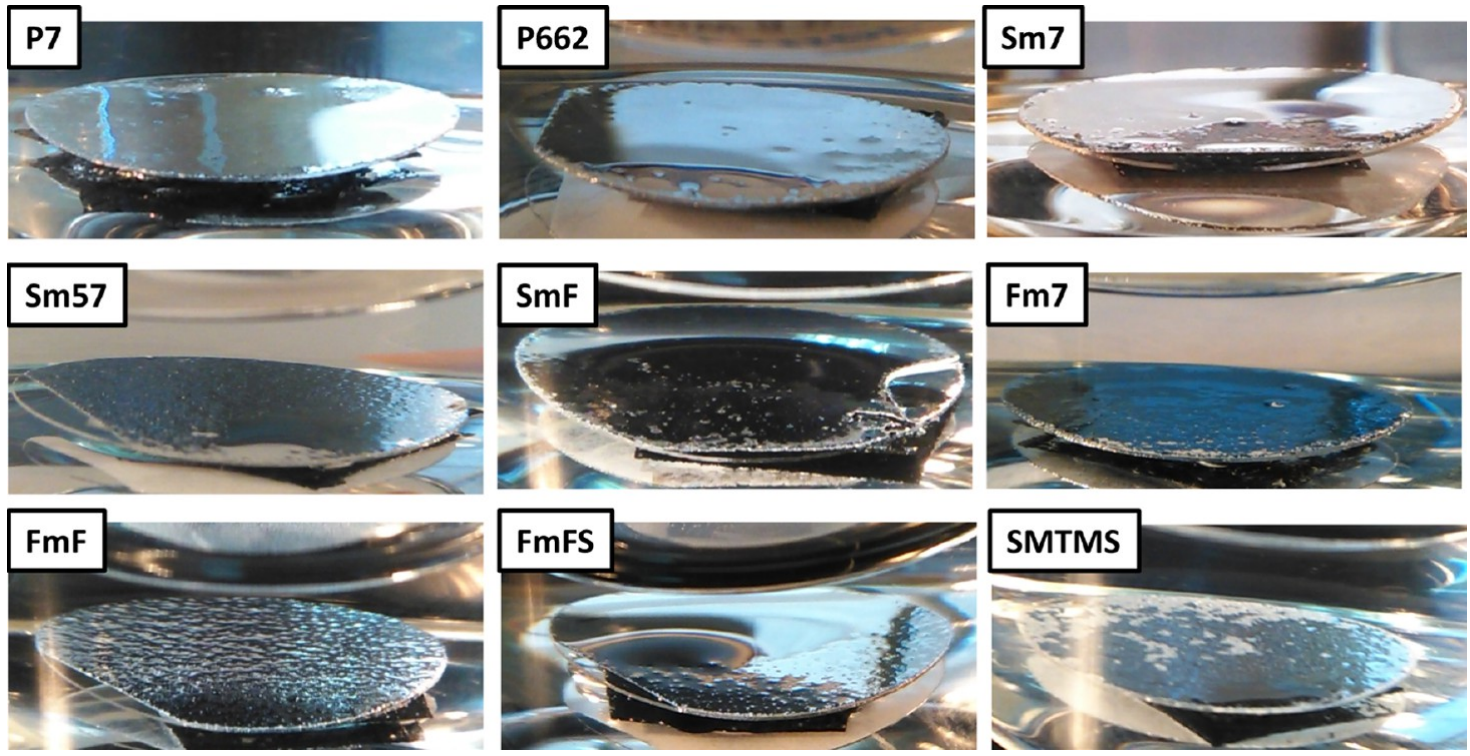

Figure 2. Pictures showing the underwater mirror effect of some of the coatings prepared with the synthesized particles.

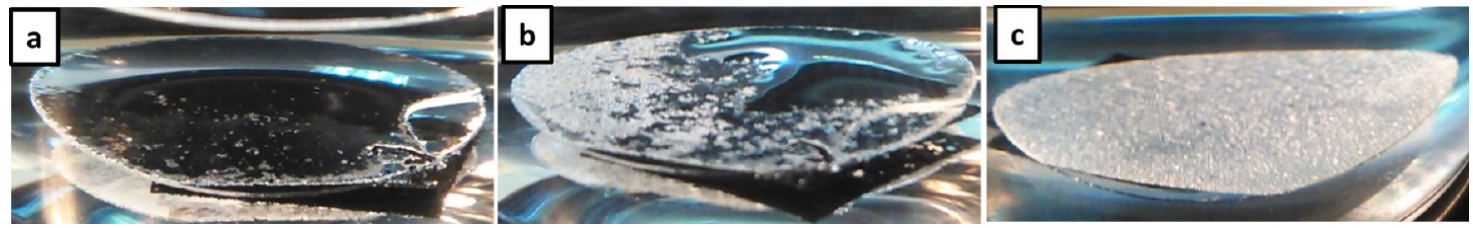

Figure 3. From left to right: Time evolution of the appearance of the mirror in the SmF coating (a) minutes after immersion, (b) $28 \mathrm{~h}$ after immersion, and (c) $>50 \mathrm{~h}$ after immersion.
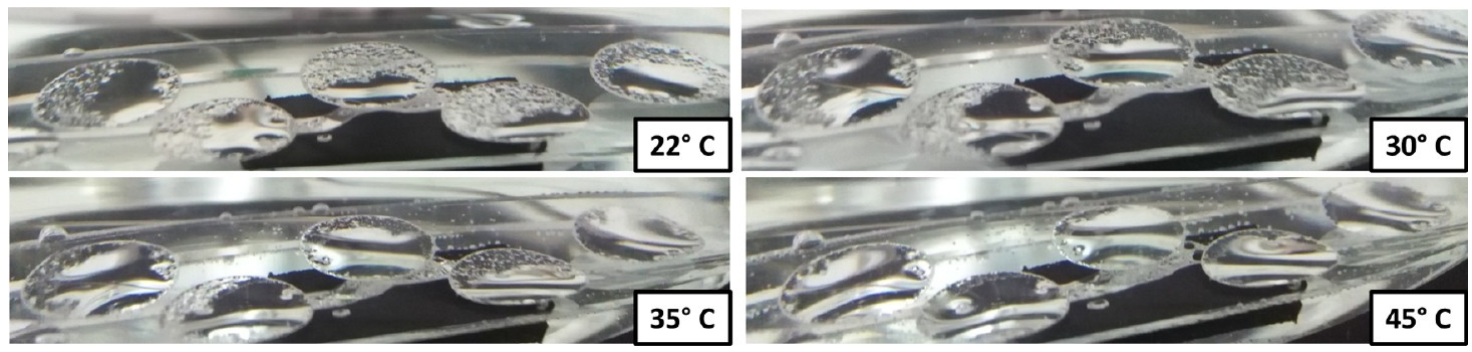

Figure 4. Evolution with water temperature of the mirrors on the coatings prepared with FmFS particles. Five circular coatings were sprayed on a microscope slide with the aid of a mask to check reproducibility of the experiments.
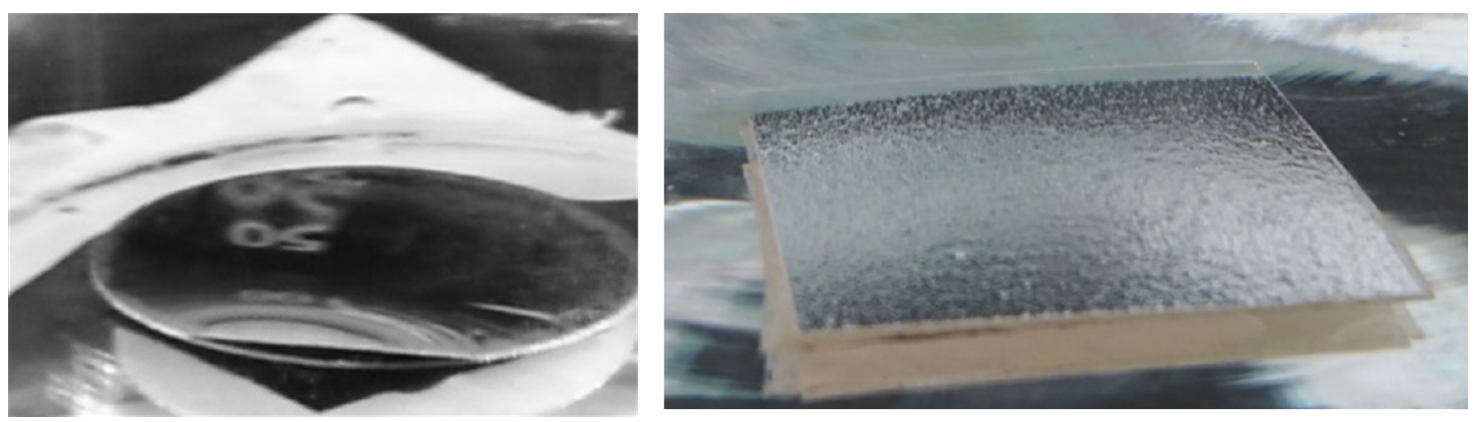

Figure 5. Effect of the substrate geometry (left: $1 \mathrm{~cm}$ diameter circle, right: $2 \mathrm{~cm}$ side square) on the quality of the mirror effect on coatings prepared with FmFS particles.

container, and the experimental parameters would not be well defined.

It is easy to see that the duration of plastrons depends very strongly on the temperature at which the experiment is done, and small variations of a couple of degrees can lead to strong differences in plastron lifetime. However, this is often not controlled (or not reported) in the results appearing in the literature. Figure 4 shows the evolution with temperature of the 

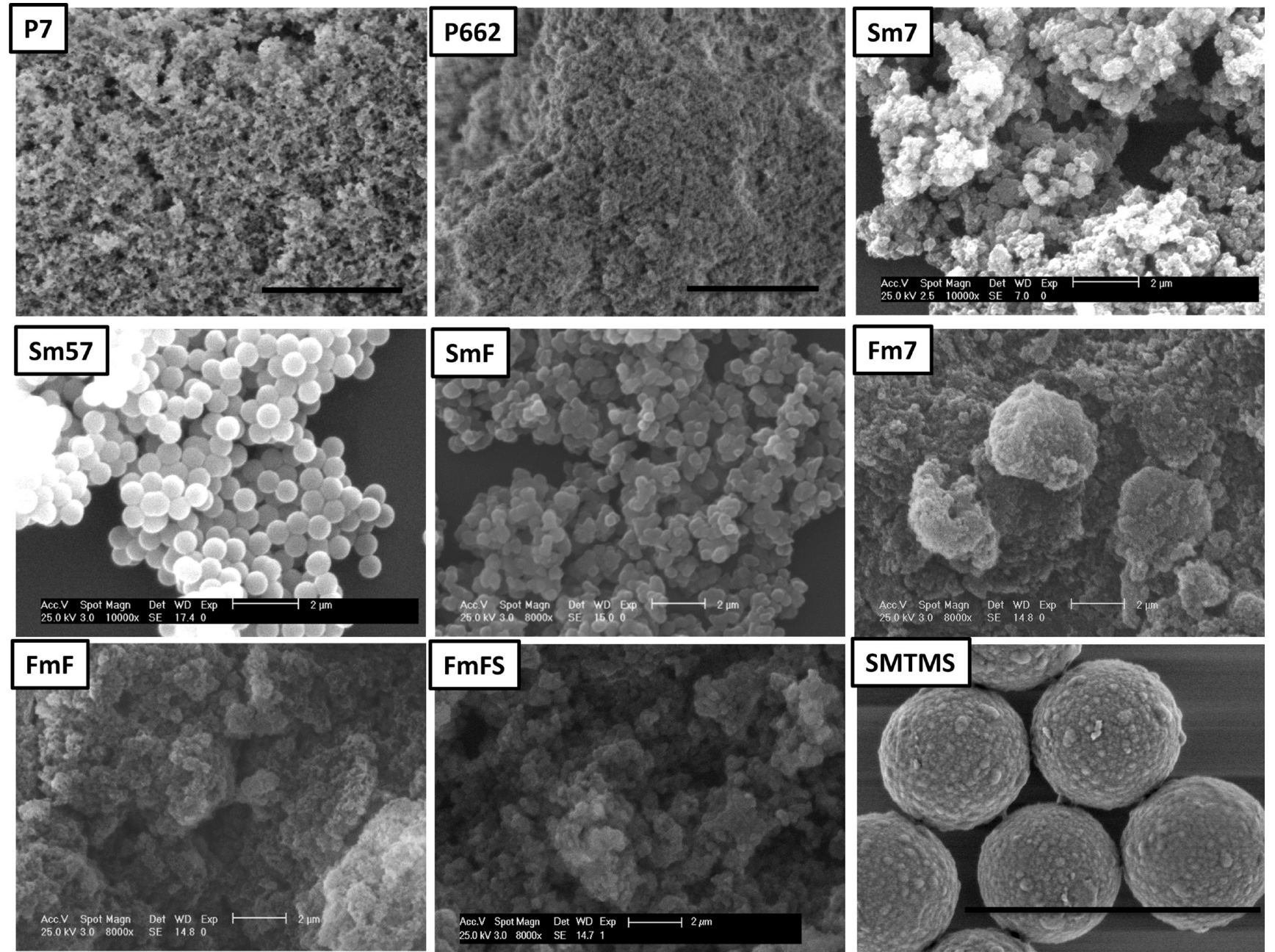

Figure 6. SEM images of some of the synthesized particles (scale bars account for $2 \mu \mathrm{m}$ ).

mirrors on the coatings prepared with FmFS particles. To check reproducibility of the properties, five circular coatings were sprayed onto a microscope slide. It can be clearly observed that increasing the water temperature concomitantly improves the extension of the reflective surface, from a section of the circular coating at $22{ }^{\circ} \mathrm{C}$ to the whole coating at $45{ }^{\circ} \mathrm{C}$. This is not surprising as temperature affects the solubility of the gases dissolved in water, the air layer volume, and the release of occluded air in the coatings to the air layer, all of which affect the amount of trapped air.

We have also checked the effect of the coating geometry (in our case, substrate) on plastron lifetime, which, to the best of our knowledge, has not been done before. It was found that mirrors are significantly better developed on circular substrates than on square or rectangular substrates. Figure 5 compares the mirror effect on circular and square substrates coated with FmFS particles. It can be seen that on the circular substrate, it is possible to see the reflection of the number scale on the beaker that contains the substrate, implying the formation of a large primary plastron, whereas the silver shine on the square substrate and the lack of a mirror-like reflection indicates the formation of many air caps and possibly water repellency, but not of a primary plastron.

The considerations above led to the experimental setup described in the Experimental Section: a temperaturecontrolled experiment performed on circular substrates of the same size enduring a small $2 \mathrm{~cm}$ water column. These experiments reveal that there is no direct relationship of the mirror lifetimes collected in Table 1 with either the type of modifier or the OM\%. This suggests that, within the set of samples selected for this study, the chemical nature is not ultimately responsible for the underwater behavior of the coatings. For the samples in this work, all of them being superhydrophobic, the key point seems to be rather the morphology of the surface of the coating, which can be visualized by scanning electron microscopy (SEM) imaging.

Figure 6 gathers SEM images of the surfaces of the coatings prepared from some of the particles collected in Table 1. At this scale, it can be seen that the morphologies are quite diverse: P7, P662, and PFS (not shown but indistinguishable from P7) are sponge-like agglomerate nanometric structures of identical appearance as the original A200 particles. ${ }^{17} \mathrm{Sm} 7, \mathrm{Fm} 7, \mathrm{FmF}$, FmFS, and SmFS (not shown but indistinguishable from the rest of this subgroup) are also constituted by nanometric structures consisting of shapeless particles, but differing in their aggregate size, and SMTMS and Sm57 are spherical particles with and without nanotexture on the surface.

These images, together with the aggregate size listed in Table 1 , give a realistic and useful description of the particles. Organosilicas derived from the modification of A200 (P7, P662, and PFS) are the most representative examples of soft silicas. P7 and PFS organize themselves into the smallest and less 
polydisperse (low Pdi) soft aggregates, both show the same morphology in SEM imaging, and they only differ in the chemical nature of the modifier. P662 is morphologically similar to these ones, but it organizes into much larger aggregates. Actually, P662 forms the biggest aggregates of the particles listed in Table 1. Sm57 and SMTMS are discrete and hard spherical microparticles, homogeneous in size (low Pdi), the latter bigger than the former. The rest of the particles, i.e., those nondiscrete particles prepared by chemisorption, are of similar morphology according to SEM imaging, with aggregates of very heterogeneous sizes according to DLS (Pdi values close to or higher than 0.30$)$. Their synthesis procedure and characterization suggest that these aggregates are not as hard as the spherical particles Sm57 and SMTMS, or as soft as P7 and PFS. It must be pointed out that the aggregate size of FmFS is the smallest of this group, and its Pdi is among the smallest, and hence it is the one that most resembles the physisorbed P7 and PFS.

It is noteworthy that this classification of the organosilica particles/aggregates into three groups, i.e, hard and large, soft and large, soft and small, turns out to be clearly related to the duration of the plastron in coatings prepared with them. As shown in Table 1 , the coatings prepared with hard, spherical particles (SMTMS and SM57) exhibit the shortest mirror lifetimes, regardless of their different chemical nature and surface morphology. Coatings prepared with softer aggregates of similar or even much larger sizes (e.g., Sm7, FmF, and P662) exhibit plastrons with much longer lifetimes than those of SMTMS or SM57. Finally, the longest lifetimes are associated with the smallest and less polydisperse soft aggregates: P7 > FmFS > PFS; P7 being, by far, the best performing particle to sustain a long-lasting air layer on its surface.

Once this direct connection between the size and polydispersity of the aggregates/particles and the mirror lifetimes under immersion is known, the next step is to find out how these parameters (softness, size, and polydispersity) are transferred into the coating surface where the plastron develops. To do that, a thorough study of the coating topographies was done. Two different techniques were employed. Atomic force microscopy (AFM) analysis is suitable for nanometric length scales (up to $2 \times 2 \mu \mathrm{m}^{2}$ ), but because of the strong topography (peak-valley distance $>1 \mu \mathrm{m}$ ) of the samples, optical profilometry was performed to cover higher scales, and the micrometer (or rather submacroscopic) scale $\left(120 \times 150 \mu \mathrm{m}^{2}\right)$ was studied by employing the highest optical objective available $(100 \times)$. For some of the coatings, it was impossible to obtain appropriate AFM images even at the $2 \times 2$ $\mu \mathrm{m}^{2}$ scale. Table 2 collects the results obtained after the analysis of the topographic data for some representative coatings.

Figure 7 collects some examples of AFM cross sections and three-dimensional (3D) images to better illustrate the roughness of the coatings. This figure and the data in Table 2 illustrate the differences in roughness profile of the coatings. Those previously defined as hard silicas (the spherical Sm57 and SMTMS) present maxima in $\mathrm{Rq}$ and $f$ when the analyzed topographic scale is in the range of the particle diameter (see Figure 7 and Table 2), whereas for soft silicas, the coating topography is not solely related to the aggregate size but also depends on its polydispersity and on the aggregate softness, all of which determine the entities' ability to pack (and to coat). Assuming that the topography at the nanoscale can be estimated by the roughness factor, $f,{ }^{30}$ it can be seen that P7, $\mathrm{P} 662$, and $\mathrm{Fm} 7$ present the largest $f 0.2$ values (it can be

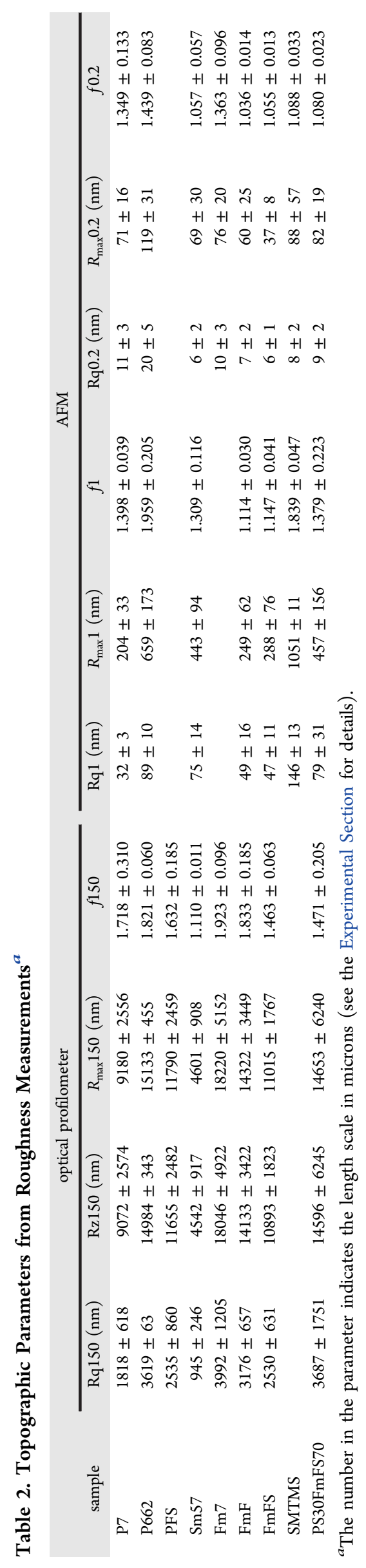




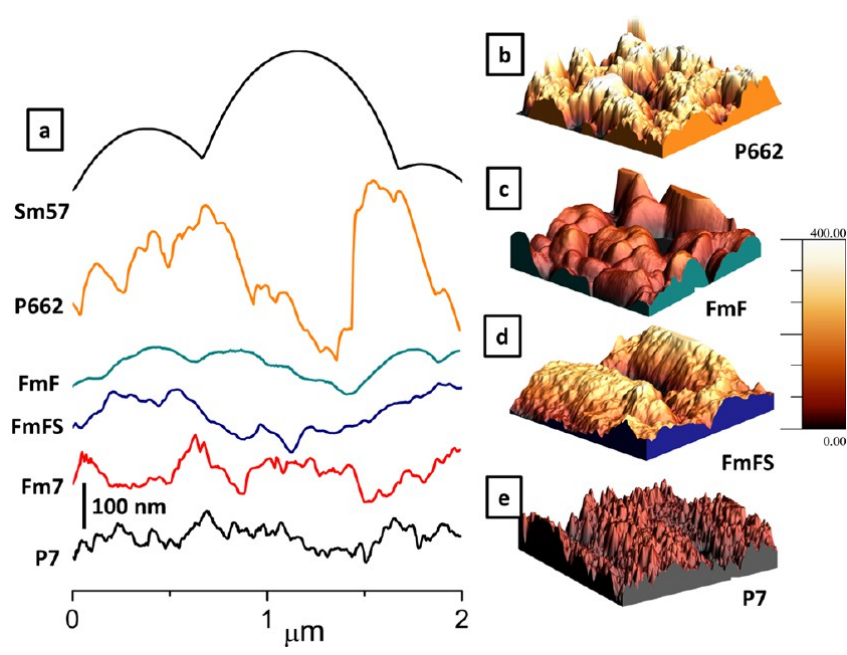

Figure 7. (a) Two micrometer AFM profiles of different coatings and 3D AFM images $\left(2 \times 2 \mu \mathrm{m}^{2}\right)$ of (b) P662, (c) FmF, (d) FmFS, and (e) P7. The color scale in the 3D images is the same for all of them and goes up to $400 \mathrm{~nm}$ high.

supposed that the PFS coating coming, as with P7 and P662, from the modification of A200 silica would exhibit a similar $f$ value, however, it was impossible to measure it by AFM). However, at this scale, both this factor, $f$, and $\mathrm{Rq}$, which are highest for P662, do not seem to have anything to do with the mirror lifetimes trend. Both $\mathrm{Rq}$ and $f$ at nano- and microscales are typically related to superhydrophobicity, and as a matter of fact, the highest water contact angle is that of P662. It is also true that FmFS, which displays one of the most stable plastrons, has one of the lowest water contact angles $\left(160^{\circ}\right)$. It seems that although a certain degree of roughness is required to attain sufficient hydrophobicity for the plastron to be produced, over this threshold, it is not higher roughness that will make the plastron last longer.

Observation of the topography at a larger scale of $1 \mu \mathrm{m}$ shows that P7, the coating with the longest mirror lifetime, is the smoothest one, with the lowest $\mathrm{Rq} 1$ and $R_{\max } 1$ (see Figure 7 ), and this is the first indication of a likely influence on plastron lifetime. At the larger $150 \mu \mathrm{m}$ scale, the effect of topography smoothness on visually detectable plastron duration is even clearer. Actually, the Rq150 values obtained from the analysis of the profilometer data reveal an inverse dependence with plastron lifetime; the lower the former, the longer the latter. In fact, $R_{\max } 150$ is the parameter that better fits with the mirror lifetimes, and the dependency is represented in Figure 8. It can be observed that when increasing the $R_{\max }$ of the coatings, the mirror lifetimes decrease. Given that in this work, we are studying large visually detectable plastrons, i.e., plastrons occurring in the macroscopic scale, it is not surprising that an inverse dependence of their duration with the $R_{\max }$ at submacroscopic scale is found. Figure 8 also shows that not all of the organosilicas represented fit the same dependency, and Sm57, which has a very low $R_{\max }$ at $150 \mu \mathrm{m}$, displays the plastron for a very short time (Sm57 appears at the lower right hand side corner of the graph). This illustrates that another requirement for long-lasting plastrons to develop is a minimum roughness, which can be characterized by a sufficiently high roughness factor $f$ or by $\mathrm{Rq}$ at submacroscopic scales. Sm57 presents a very low $f 150$ value $(f=1.1)$ at this scale (far larger than its particle diameter), and it cannot be fitted in the trend

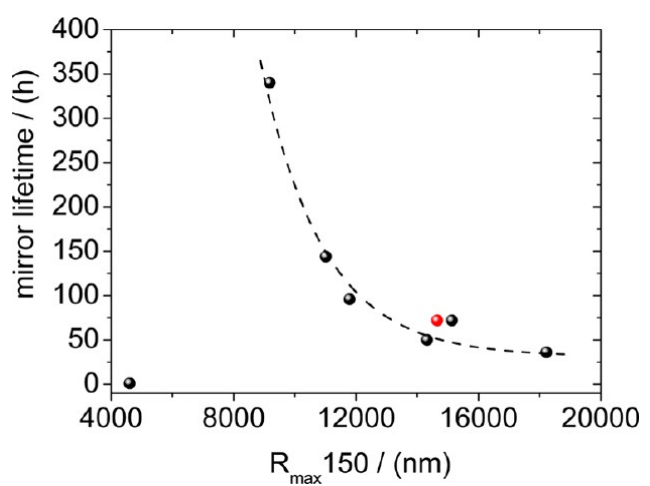

Figure 8. Mirror lifetimes vs $R_{\max } 150$ for the particle coatings in Table 2. The particle-polymer coating PS30FmFS70 is the red point. The dotted line is a mere guide for the eyes.

depicted in Figure 8 for the soft organosilicas, all of which have $f 150 \geq 1.5$.

SEM images of coating sections of P7, PFS, Fm7, and FmFS are presented in Figure 9. This figure also contains $150 \mu \mathrm{m}$ cross-sectional data as obtained by optical profilometry of the same coatings. The comparison of these coatings is very interesting because (i) Fm7 particles are similar in size to P7, PFS, or FmFS, (ii) the $\mathrm{Fm} 7$ coating presents a similar value of $f 0.2$ as that of $\mathrm{P} 7$, showing nanometric roughness (also assumed in PFS), not detected in FmFS, (iii) roughness parameters at the long scale $(150 \mu \mathrm{m})$ are significantly larger for $\mathrm{Fm} 7$ than for the other three coatings, and (iv) the mirror lifetime in $\mathrm{Fm} 7$ is four times shorter than that in FmFS and about 10 times shorter than that in P7. The SEM images show that P7, PFS, and FmFS present regular sections, as observed by SEM and optical profilometry, with $R_{\max } 150$ values of around $10 \mu \mathrm{m}$. In contrast, $\mathrm{Fm} 7$ presents an inhomogeneous section with large agglomerations that can be over $20 \mu \mathrm{m}$ in height. In the light of this evidence and as the chemical nature does not play an important role in the mirror effect of this set of particles (P7 and $\mathrm{Fm} 7$ are actually synthesized using the same modifier), the conclusion drawn is that the $\mathrm{Fm} 7$ particles are more difficult to pack into a regular and "smooth" coating, and this is the reason behind the short duration of the mirror effect in this coating. The "packing" (or "coating") ability of the particles will rely not only on the aggregate size but also on its polydispersity, which is notably larger in $\mathrm{Fm} 7$ compared to that of the other three particles in Figure 9. Fm7 particles are very heterogeneous in size, and this fact is transferred into a steep roughness profile ${ }^{22}$ (even visualized as gaps in the coating section in the SEM image of Figure 9), which impedes the formation of a long-lasting plastron.

Bearing in mind the physical process behind the mirror effect as studied in this work, namely, the eye visualization of a continuous mirror formed by the air plastron, it is quite obvious that the control parameter is $R_{\max }$ rather than $\mathrm{Rq}$. The higher the $R_{\max }$ values, the thicker the plastron required to visualize a homogeneous mirror, and the sooner the primary plastron will lose continuity as the air layer becomes thinner because of water pressure. The air plastron height required to develop a continuous mirror on the surface coating is depicted in the cross sections in Figure 9. Once the plastron is not continuous, the mirror becomes deteriorated until it is completely lost. This finding demonstrates that, given a superhydrophobic surface (which requires $f$ or $\mathrm{Rq}$ to be over a minimum threshold), the key for a long-lasting primary plastron is not a very high 

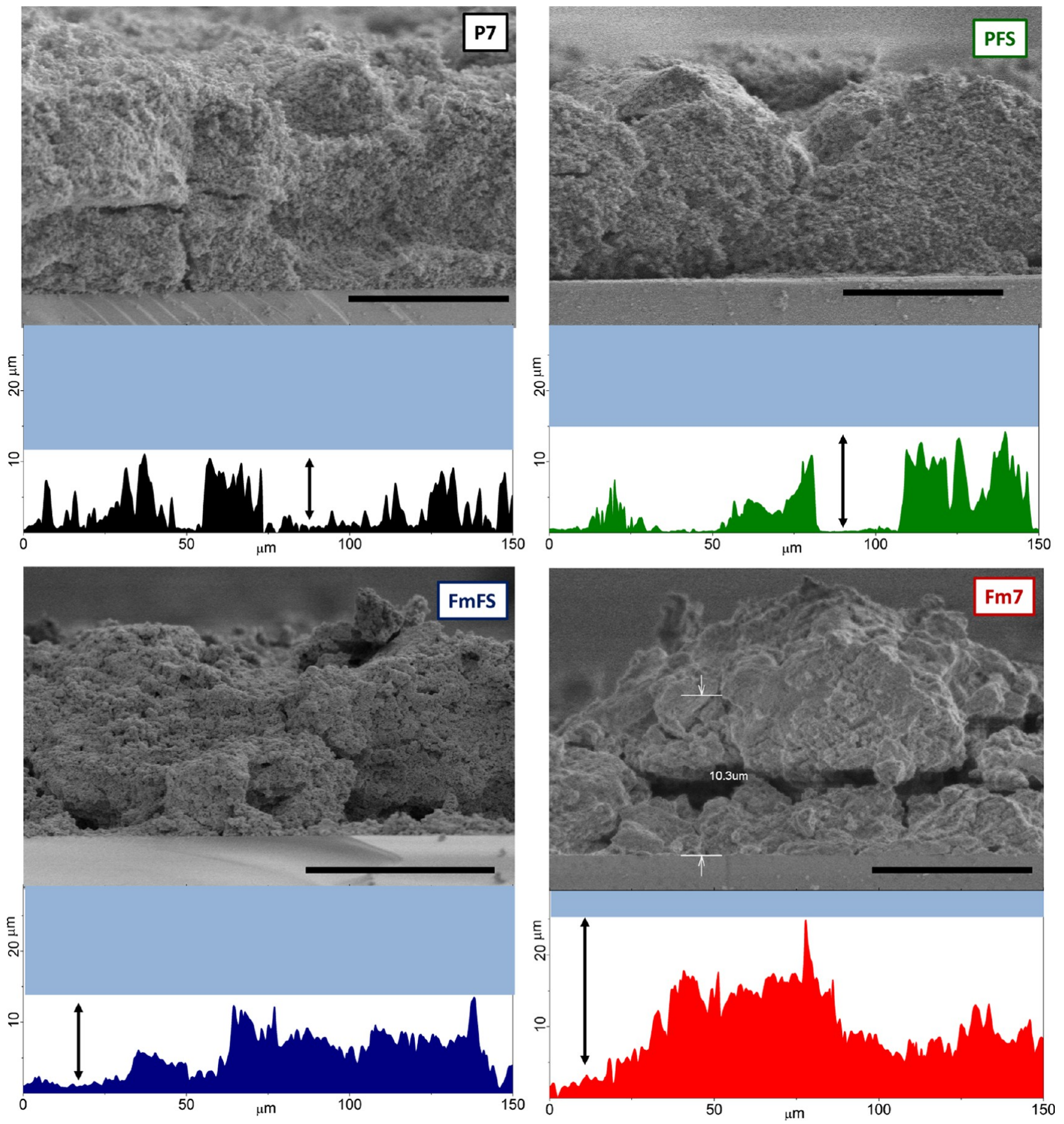

Figure 9. SEM images of sections of some coatings (scale bar is $10 \mu \mathrm{m}$ ). One representative cross section obtained from optical profilometry for the same coating is under each SEM image. The air layer height required for the development of the mirror effect (primary plastron) is depicted in the cross sections.
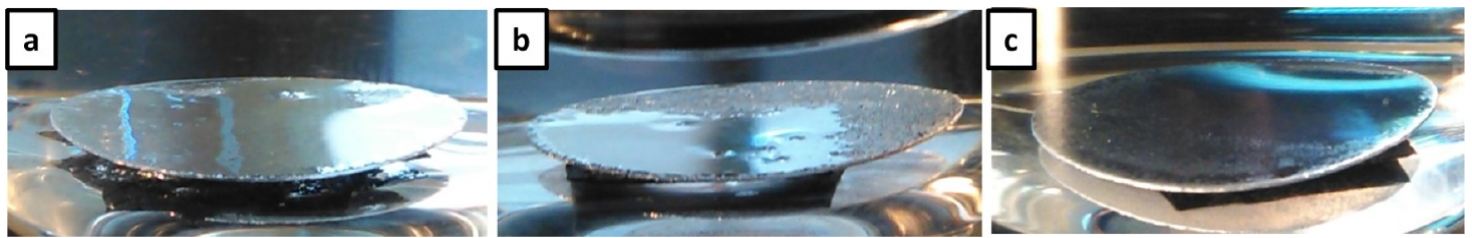

Figure 10. Pictures showing the appearance of the mirror on coatings prepared with P7 particles: (a) minutes after immersion, (b) after $100 \mathrm{~h}$ of immersion, and (c) after removing the water and filling the container again. 


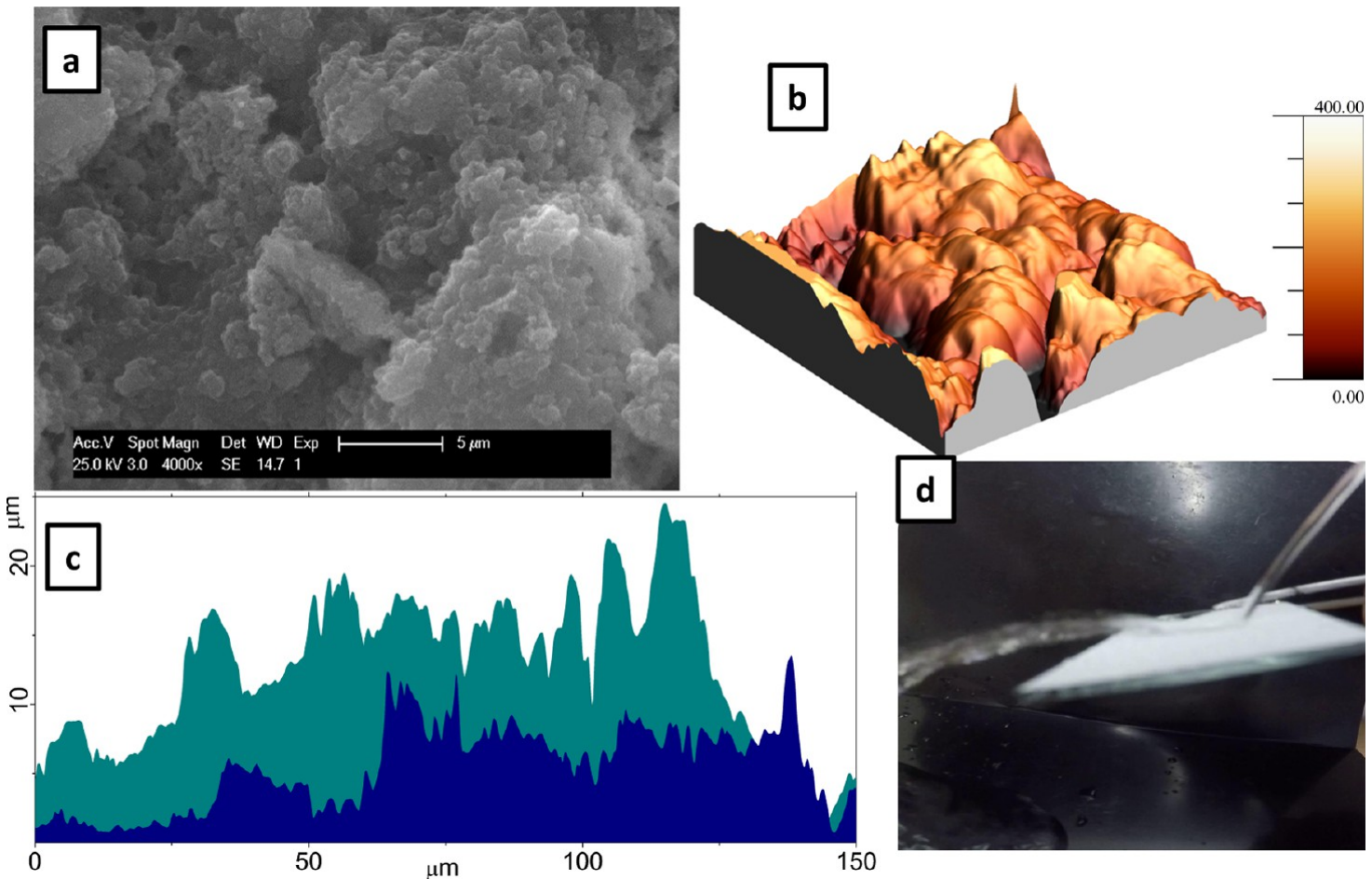

Figure 11. a) SEM image and (b) 3D AFM image $\left(2 \times 2 \mu \mathrm{m}^{2}\right)$ of the coating PS30FmFS70, (c) cross sections obtained from optical profilometry of this coating (in dark cyan) compared to the coating from the FmFS particles (in blue), and (d) picture showing the behavior with water of the coating.

roughness factor, as the theory suggested, ${ }^{31}$ but a homogeneous and relatively smooth topography.

The question remains as to whether the primary plastron is reversible once it is lost or, in other words, what happens to the hydrophobicity of the surface when the mirror/air plastron disappears. To shed some light on this important issue, we checked that in the samples studied in this work, the mirror effect is a reversible event, meaning that after the mirror is lost or deteriorated, it can be recovered by exposing the coating to air and filling again with water. One example is shown in the pictures of Figure 10. Figure 10a is a picture of the mirror on a coating of P7 particles just after filling the water column. Figure $10 \mathrm{~b}$ shows the same coating after $100 \mathrm{~h}$ under immersion. The mirror is still there but it does not cover the whole coating surface. Figure 10c shows the appearance of the same coating after removing the water column and immediately filling again with fresh water. The mirror appears to be homogeneous and undistinguishable from that in Figure 10a. Therefore, the coating is still able to sustain an air layer even after losing or partially losing the mirror. This implies that the plastron (or mirror) is lost in these cases because of the decreasing of the air layer thickness and not because of wetting (not even partial) of the surface.

In this regard, we have checked that coatings prepared with P7, PFS, or FmFS can be underwater for months (in the case of P7, at least 6 months) and still be superhydrophobic immediately after removing the water, without drying the coating.

Therefore, it can be stated that although the presence of a primary plastron is an indication of a very hydrophobic underwater surface, the disappearance of the primary plastron after a given time is not an indication of the lack of superhydrophobicity of a surface exposed to water. This has important consequences, and it makes the design of new experiments to estimate the persistence of underwater hydrophobicity necessary. This is important not only for academic purposes, but also to evaluate the adequacy of given materials for specific applications.

The process of removing the water column and filling it again (to check the formation of a new mirror or to check the hydrophobicity of the surface after immersion) requires robust coatings that do not suffer damage after several cycles. This is definitively not the case for coatings prepared only with organosilica particles and no binder. A way to solve this problem would be to use double sided tape to stick the coating to the substrate, as reported in a recent study, ${ }^{32}$ which is rather limited as regards applicability, or our preferred option, which is the mixture of the particles with a polymer matrix acting as a particle binder. ${ }^{17}$ In this particular case, the FmFS particles were mixed with polystyrene at a weight ratio of 30:70 (PS/ FmFS). The last column of Table 2 and Figure 11 collect some information on this coating.

The results show that after mixing with the polymer, the roughness parameter $R_{\max }$ of the coating at $150 \mu \mathrm{m}$ increases, and concomitantly, there is a decrease in mirror lifetime compared to that of the only-particle coating. The mirror lifetime of the PS30FmFS70 coating is around $72 \mathrm{~h}$ and its plastron lifetime follows the trend shown in Figure 8, where PS30FmFS70 appears plotted in red. Therefore, even if PS30FmFS70 incorporates a non-negligible amount of polymer binder, as regards plastron lifetime, it belongs to the same family of coatings as the rest of the soft organosilicas in Figure 8 (contrary to what happens to the hard organosilica Sm57), and indeed its roughness factor is $f 150 \approx 1.5$, like other members of the family. Together with a very remarkable underwater hydrophobicity, PS30FmFS70 has good mechanical stability and can be handled without risk of damage (see Figure 11d and 
Scheme 1
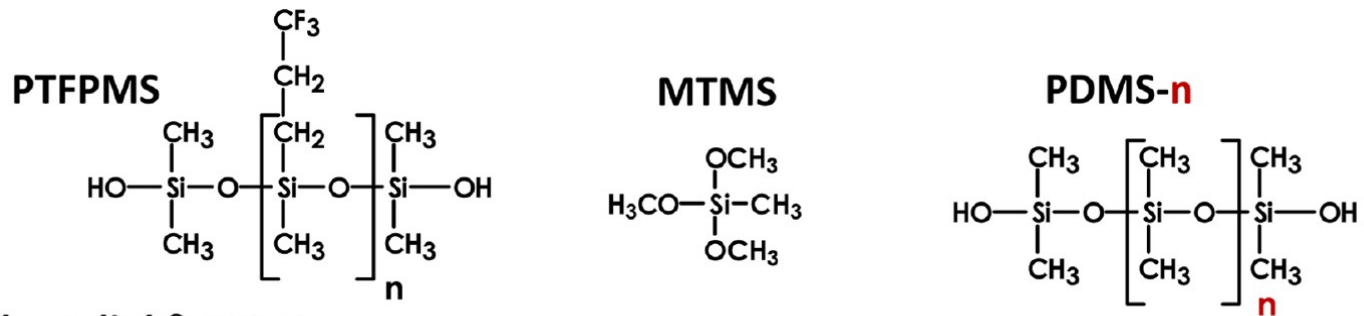

Fluorolink $^{\circledR}$ E10-H

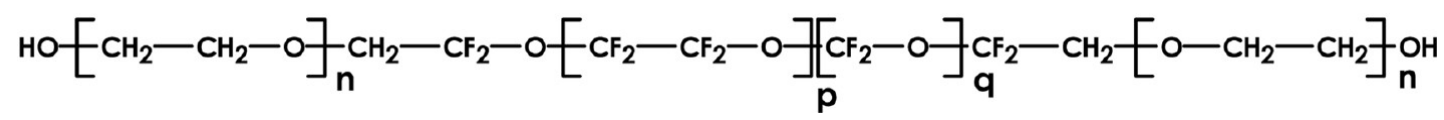

Movie, SI), and it represents a very promising approach to long-lasting robust underwater superhydrophobic materials.

\section{CONCLUSIONS}

The underwater hydrophobicity of a family of hybrid organicinorganic silica-based particle coatings has been studied, and in particular, their ability to develop primary plastrons under immersion, a continuous air layer occluded between the coating surface and the water column. The family includes soft, pliable organosilicas derived from the physisorption of siloxane and fluorosiloxane chains, hard spherical organosilicas, and other organosilicas in between those two limit morphologies. These particles have been used to prepare circular superhydrophobic coatings by spraying. The topographic parameters at the nanoand microscales have been measured, and underwater hydrophobicity endurance has been tested using a shallow $2 \mathrm{~cm} 20$ ${ }^{\circ} \mathrm{C}$ water column. A primary plastron, characterized by the socalled mirror effect, appears in all of the samples. Strong effects of the water temperature and the geometry of the coating on the endurance of the primary plastron were detected, whereby plastrons developed much better at higher temperatures and in circular coatings. Once the lifetime of the plastron was studied by well controlled experiments, it was possible to detect, within this family of organosilicas, a strong dependency of its duration $(\times 350 \mathrm{~h})$ on the smoothness of the coatings, as characterized by the lowest $R_{\max }$ and $\mathrm{Rz}$ values in surfaces of $120 \times 150 \mu \mathrm{m}^{2}$. The air plastrons prove to be reversible when the water column is removed and immediately refilled, which evidences that superhydrophobicity remains after the loss of primary plastrons. Furthermore, it was checked that some of the coatings remain superhydrophobic after 6 months underwater immersion. A polymer composite with polystyrene and one of the particles was prepared, and it was shown that it behaves in the same way as the particle-only coatings, exhibiting a durable primary plastron together with remarkable mechanical stability.

To sum up, this work provides useful experimental insights for the optimal design of long-lasting underwater superhydrophobic surfaces required in several application fields of superhydrophobicity.

\section{EXPERIMENTAL SECTION}

Materials. Hydrophilic fumed silica, A200, kindly supplied by Degussa, silanol-terminated oligodimethylsiloxane of 7,57 , and 662 repeat units as average, PDMS-7, PDMS-57, and PDMS-662, respectively, silanol-terminated poly(trifluoropropylmethylsiloxane) (PTFPMS) from ABCR, Fluorolink E10-H from Solvay, methyltrimethoxysilane (MTMS), TEOS supplied by Sigma-Aldrich, and polystyrene (PS,
Styrolution $168 \mathrm{~N}$ ) were all used without further purification. Solvents for reactions, filtration, and chromatography were certified ACS grade and were purchased from Sigma-Aldrich.

Scheme 1 collects the chemical structure of the surface modifiers and comonomers in the condensation reactions.

Preparation of Organosilicas. Surface Modification. The surface modification of Aerosil 200 was done as reported in reference. ${ }^{17}$ Basically, $3 \mathrm{~g}$ of A200 and $1.76 \mathrm{~g}$ of PDMS- $n(n=7$ or 662 ) or PTFPMS in $200 \mathrm{~mL}$ of toluene were magnetically stirred for $72 \mathrm{~h}$. The resulting organosilicas, $\mathrm{P} n(n=7$ or 662$)$ or PFS (PTFPMS as surface modifier), were isolated from the reaction medium by centrifugation and purified by means of repetitive washing in dichloromethane and acetone (three times) and further centrifugation. Finally, materials were dried at $100{ }^{\circ} \mathrm{C}$ overnight.

Condensation Reactions. Two different synthetic approaches were carried out to prepare the organosilica by condensation reactions: the conventional Stöber method ${ }^{33}$ and a fluoride-catalyzed condensation. ${ }^{34}$

In the sol-gel Stöber method, $3 \mathrm{~mL}$ of milli-Q $\mathrm{H}_{2} \mathrm{O}, 22 \mathrm{~mL}$ of ethanol, and $3 \mathrm{~mL}$ of $\mathrm{NH}_{4} \mathrm{OH}$ were placed in a three-necked flask equipped with a mechanical stirrer. After $30 \mathrm{~min}$ of stirring, the appropriate quantities of the organic modifier and 3 $\mathrm{mL}$ of TEOS were added. The mixture was magnetically stirred for another $21 \mathrm{~h}$ at $20{ }^{\circ} \mathrm{C}$. Then, the gel was recovered by centrifugation, and washed with abundant distilled water up to $\mathrm{pH}=7$. Finally, the sample was dried in two steps, first at 100 ${ }^{\circ} \mathrm{C}$ overnight and then at $250{ }^{\circ} \mathrm{C}$ under vacuum for $7 \mathrm{~h}$. Following this method, four organosilicas were prepared: $\mathrm{Sm} 7$ (with $0.47 \mathrm{~g}$ of PDMS-7), Sm57 (with $2.68 \mathrm{~g}$ of PDMS-57), SmF (with $0.47 \mathrm{~g}$ of Fluorolink), and SmFS (with $0.47 \mathrm{~g}$ of PTFPMS). For the synthesis of Sm57, isopropanol was used as solvent instead of ethanol.

For the fluoride-catalyzed condensation, an initial mixture of TEOS $(3 \mathrm{~mL})$, ethanol $(4.7 \mathrm{~mL})$, and the comonomer $(0.47 \mathrm{~g})$ was mechanically stirred for $30 \mathrm{~min}$. After that, an aqueous solution of $\mathrm{NH}_{4} \mathrm{~F}(0.15 \mathrm{~g}$ in $1.5 \mathrm{~mL})$ was slowly added to the organosilicon solution. The mixture was stirred at room temperature until the gelation took place (approx. $1 \mathrm{~h}$ ). Then, the gel was kept aging at $36{ }^{\circ} \mathrm{C}$ for $24 \mathrm{~h}$, and finally dried at $150{ }^{\circ} \mathrm{C}$ under vacuum for another $24 \mathrm{~h}$. Three organosilicas were synthesized by this method: Fm7 (with PDMS-7 as comonomer), FmF (with Fluorolink as comonomer), and FmFS (with PTFPMS as comonomer).

Finally, the organosilica SMTMS was prepared by a selfcondensation reaction of MTMS. MTMS (0.55 g) was dissolved in $15 \mathrm{~mL}$ of $\mathrm{H}_{2} \mathrm{O}$ milli-Q and $0.025 \mathrm{~mL}$ of $\mathrm{NH}_{4} \mathrm{OH}$ was added to the solution. The reaction mixture was 
kept at $20{ }^{\circ} \mathrm{C}$ for $24 \mathrm{~h}$. Then, the particles were isolated by centrifugation, washed with water up to $\mathrm{pH}=7$, and dried at $100{ }^{\circ} \mathrm{C}$ for $24 \mathrm{~h}$.

Preparation of the Particle Coatings. Organosilica suspensions in ethanol at a concentration of $27 \mathrm{mg} \mathrm{mL}$ were stirred at room temperature for $24 \mathrm{~h}$ before the deposition on glass supports by spraycoating with an airbrush AB931 from Sealey. Two different glass supports were employed, circular glass supports of $1 \mathrm{~cm}$ diameter, and microscope slides onto which circular coatings were sprayed with the aid of a mask. This was done to check the reproducibility of the measurements. No significant differences in $t_{\text {mirror }}$ were found between these two types of circular coatings. Square glass slides of $2 \mathrm{~cm}$ side were used to check the influence of the substrate shape on the development of the mirror underwater immersion.

Preparation of the Polymer-Particle Coating. The desired amount of FmFS particles to obtain a 70 wt \% blend was added to a previously prepared PS solution in toluene $(2.5$ wt \%). The mixture was stirred for $3 \mathrm{~h}$ and then sprayed onto glass supports with the airbrush.

Characterization of the Organosilica and Coatings. The organic mass (OM\%) was calculated from carbon content (C\%) obtained by LECO CHNS-932 equipment, with an error of $\pm 0.3 \%$.

Solid NMR spectroscopy was performed on a Bruker AvanceTM 400 spectrometer (Bruker Analytik GmbH Karlsrube, Germany) equipped with a Bruker UltrashieldTM $9.4 \mathrm{~T}\left({ }^{29} \mathrm{Si}\right.$ frequency of $\left.79.49 \mathrm{MHz}\right), 8.9 \mathrm{~cm}$ vertical-bore superconducting magnet. In both cases, ${ }^{13} \mathrm{C}$ and ${ }^{29} \mathrm{Si}, \mathrm{CP} / \mathrm{MAS}$ NMR spectra were acquired at ambient temperature by using a standard Bruker broad-band MAS probe. Representative samples were ground and packed in $4 \mathrm{~mm}$ zirconia rotors, sealed with Kel-FTM caps, and spun at $5 \mathrm{kHz}$. The $90^{\circ}$ pulse width was $3.5-4.5 \mu \mathrm{s}$ and, in all cases, high-power proton decoupling was used. All free-induction decays were subjected to standard Fourier transformation and phasing. The chemical shifts were externally referenced to tetramethylsilane.

The particle aggregate size, $Z_{\text {ave }}$ and the polydispersity index, Pdi, were evaluated by dynamic light scattering (DLS) with a Zetasizer Nano ZS (Malvern Instruments) on suspensions in ethanol with a particle concentration of $50 \mu \mathrm{g} \mathrm{mL}^{-1}$. The values reported are the mean values of three measurements.

The surface coating morphology was visualized by scanning electron microscopy (SEM) using a Philips XL30ESEM and a Hitachi SU-8000.

Atomic force microscopy (AFM) was used to examine the surface topography. The AFM images were obtained with a Veeco Multimode scanning probe microscope equipped with a Nanoscope IV a controller operating in tapping mode with a phosphorus-doped silicon cantilever (model RTESP). Topography at higher length scales were analyzed by optical profilometry using a Zeta-20 optical profiler (Zeta Instruments) with a $100 \times$ optical objective and $13 \mathrm{~nm}$ of vertical resolution. Topographic parameters, mean square roughness values, $\mathrm{Rq}$, rugosity factor, $f$, defined as real surface divided by geometric surface, $\mathrm{Rz}$, defined as the average of the absolute values of the heights of the five highest profile peaks and the depths of five deepest valleys within the evaluation length, and $R_{\max }$ defined as the largest vertical distance between the highest peak and the lowest valley in the profile, were all calculated from the cross sections or $3 \mathrm{D}$ images obtained by optical profilometry and AFM and are average values of at least five measurements. The $x$ number, in $\operatorname{Rq} x, \operatorname{Rz} x, R_{\max } x$, and $f x$, indicates the length scale (cross section or $3 \mathrm{D}$ image side) in microns.

The use of the topographic parameters above as a characterization means will only make sense in homogeneous and reproducible coatings where the topography depends on known and controlled experimental factors (in this case, solvent and concentration of the dispersion, processing aid, and particle employed) and not on other accidental circumstances. A simple test of the homogeneity of the coating consists of comparing $\mathrm{Rz}$ and $R_{\max }$, which (i) must vary in the same way in the different coatings and (ii) cannot be very different from one another.

Apparent contact angles were measured with Milli-Q grade water by the sessile drop method using a conventional drop shape analysis technique (Attension Theta optical tensiometer). For dynamic measurements (advancing and receding contact angles required to obtain $\Delta \theta$ ), the initial water drop volume was $5 \mu \mathrm{L}$ and the volume was increased up to $10 \mu \mathrm{L}$ by water disposal at $0.2 \mu \mathrm{L} \mathrm{s}^{-1}$, recording and analyzing 10 images per second during this process. A constant contact angle value is achieved, which is considered the advancing water contact angle. Then, $5 \mu \mathrm{L}$ of water was removed at the same rate following an identical analysis protocol that enables the determination of the receding contact angles. The determination of the contact angle from the captured images is done by the Young-Laplace method. The initial water contact angle in static conditions with a $5 \mu \mathrm{L}$ volume drop is considered to be $\theta_{\mathrm{w}}$. Reported contact angles values were the average of at least five measurements in different coating regions.

To evaluate the underwater hydrophobicity, the experiments were designed as follows. The particles were deposited onto 1 $\mathrm{cm}$ diameter circle glass supports by spraying from ethanol suspensions. The supports were fixed to the bottom of vials that were filled with a $2 \mathrm{~cm}$ water column. The experiments were run with Milli-Q grade water at $20{ }^{\circ} \mathrm{C}$. The water temperature was controlled throughout the experiments. In this work, we estimate the lifetimes of primary or macroscopic plastrons, namely, continuous air cushions at the interface between the surface and the water, characterized by the formation of a reflective surface (mirror effect) on top. The duration of the mirror effect $\left(t_{\text {mirror }}\right)$ has been determined visually with the aid of a camera, and corresponds to the ability of the surface to produce a clear reflection of an image. Pictures of the surface were regularly taken and they were critically analyzed. When there was no reflection on top (we eventually checked with an image), the surface turned greyish, and the experiment was considered to be over. To check the reproducibility of the experiments, glass slides with five $1 \mathrm{~cm}$ coated circles were sprayed with the aid of a mask. The above reported experiments were reproduced with these samples and it was checked that statistical errors on $t_{\text {mirror }}$ are less than $10 \%$. The main source of error in the estimation of $t_{\text {mirror }}$ is the overnight time in long-lasting experiments. In any case, this error is not significant compared to the differences in $t_{\text {mirror }}$ between samples. The figures will show visual examples of the appearance of the continuous plastron (mirror) on the surface and its time evolution.

\section{ASSOCIATED CONTENT}

\section{Supporting Information}

The Supporting Information is available free of charge on the ACS Publications website at DOI: 10.1021/acsomega.7b01717. 
Movie showing the superhydrophobicity and mechanical stability of the PS30FmFS70 coating (AVI)

\section{AUTHOR INFORMATION}

\section{Corresponding Authors}

*E-mail: ptiemblo@ictp.csic.es (P.T.).

*E-mail: ngarcia@ictp.csic.es (N.G.).

ORCID 1

Nuria García: 0000-0002-4330-3883

Notes

The authors declare no competing financial interest.

\section{ACKNOWLEDGMENTS}

The authors would like to acknowledge funding from the Spanish Ministry (MAT2016-81001-P). We are in debt to Dr. Julián Jiménez from ICV-CSIC for his support in profilometry measurements.

\section{REFERENCES}

(1) Zhang, P.; Lv, F. Y. A review of the recent advances in superhydrophobic surfaces and the emerging energy-related applications. Energy 2015, 82, 1068-1087.

(2) Tian, Y.; Su, B.; Jiang, L. Interfacial Material System Exhibiting Superwettability. Adv. Mater. 2014, 26, 6872-6897.

(3) Marmur, A.; Volpe, C. D.; Siboni, S.; Amirfazli, A.; Drelich, J. W. Contact angles and wettability: Towards common and accurate terminology. Surf. Innovations 2017, 5, 3-8.

(4) Celia, E.; Darmanin, T.; de Givenchy, E. T.; Amigoni, S.; Guittard, F. Recent advances in designing superhydrophobic surfaces. J. Colloid Interface Sci. 2013, 402, 1-18.

(5) Harpster, H. T. The Gaseous Plastron as a Respiratory Mechanism in Stenelmis quadrimaculata Horn Dryopidae. Trans. Am. Microsc. Soc. 1944, 63, 1-26.

(6) Shirtcliffe, N. J.; McHale, G.; Newton, M. I.; Perry, C. C.; Pyatt, F. B. Plastron properties of a superhydrophobic surface. Appl. Phys. Lett. 2006, 89, No. 104106.

(7) Larmour, I. A.; Bell, S. E. J.; Saunders, G. C. Remarkably simple fabrication of superhydrophobic surfaces using electroless galvanic deposition. Angew. Chem., Int. Ed. 2007, 46, 1710-1712.

(8) Sakai, M.; Yanagisawa, T.; Nakajima, A.; Kameshima, Y.; Okada, $\mathrm{K}$. Effect of surface structure on the sustainability of an air layer on superhydrophobic coatings in a water-ethanol mixture. Langmuir 2009, $25,13-16$.

(9) Bobji, M. S.; Kumar, S. V.; Asthana, A.; Govardhan, R. N. Underwater sustainability of the "Cassie" state of wetting. Langmuir 2009, 25, 12120-12126.

(10) Poetes, R.; Holtzmann, K.; Franze, K.; Steiner, U. Metastable underwater superhydrophobicity. Phys. Rev. Lett. 2010, 105, No. 166104.

(11) Forsberg, P.; Nikolajeff, F.; Karlsson, M. Cassie-Wenzel and Wenzel-Cassie transitions on immersed superhydrophobic surfaces under hydrostatic pressure. Soft Matter 2011, 7, 104-109.

(12) Vakarelski, I. U.; Chan, D. Y.; Marston, J. O.; Thoroddsen, S. T. Dynamic air layer on textured superhydrophobic surfaces. Langmuir 2013, 29, 11074-11081.

(13) Xu, M.; Sun, G.; Kim, C. J. Infinite lifetime of underwater superhydrophobic states. Phys. Rev. Lett. 2014, 113, No. 136103.

(14) Bormashenko, E. Progress in understanding wetting transitions on rough surfaces. Adv. Colloid Interface Sci. 2015, 222, 92-103.

(15) Søgaard, E.; Andersen, N. K.; Smistrup, K.; Larsen, S. T.; Sun, L.; Taboryski, R. Study of transitions between wetting states on microcavity arrays by optical transmission microscopy. Langmuir 2014, 30, 12960-12968.

(16) Prakash, S.; Xi, E.; Patel, A. J. Spontaneous recovery of superhydrophobicity on nanotextured surfaces. Proc. Natl. Acad. Sci. U.S.A. 2016, 113, 5508-5513.
(17) De Francisco, R.; Tiemblo, P.; Hoyos, M.; González-Arellano, C.; García, N.; Berglund, L.; Synytska, A. Multipurpose ultra and superhydrophobic surfaces based on oligodimethylsiloxane-modified nanosilica. ACS Appl. Mater. Interfaces 2014, 6, 18998-19010.

(18) de Francisco, R.; Hoyos, M.; García, N.; Tiemblo, P. Superhydrophobic and highly luminescent polyfluorene/silica hybrid coatings deposited onto glass and cellulose-based substrates. Langmuir 2015, 31, 3718-3726.

(19) Ribot, F.; Sanchez, C. Organically functionalized metallic oxoclusters: Structurally well-defined nanobuilding blocks for the design of hybrid organic-inorganic materials. Comments Inorg. Chem. 1999, 20, $327-371$.

(20) Schaefer, D. W.; Kohls, D.; Feinblum, E. Morphology of Highly Dispersing Precipitated Silica: Impact of Drying and Sonication. J. Inorg. Organomet. Polym. Mater. 2012, 22, 617-623.

(21) Tsantilis, S.; Pratsinis, S. E. Soft- and hard-agglomerate aerosols made at high temperatures. Langmuir 2004, 20, 5933-5939.

(22) García, N.; Benito, E.; Tiemblo, P.; Hasan, M. M. B.; Synytska, A.; Stamm, M. Chemically Guided Topography in Alkylsilane- and Oligosiloxane-Modified Silica Nanoparticle Coatings: From very Hydrophobic Surfaces to "Pearl" Bouncing Droplets. Soft Matter 2010, 6, 4768-4776.

(23) Babonneau, F. Hybrid Siloxane-Oxide Materials via Sol-Gel Processing: Structural Characterization. Polyhedron 1994, 13, 11231130.

(24) Zhang, X. X.; Xia, B. B.; Ye, H. P.; Zhang, Y. L.; Xiao, B.; Yan, L. H.; Lv, H. B.; Jiang, B. One-step sol-gel preparation of PDMS-silica ORMOSILs as environment-resistant and crack-free thick antireflective coatings. J. Mater. Chem. 2012, 22, 13132-13140.

(25) Zhang, X.; Ye, H.; Xiao, B.; Yan, L.; Lv, H.; Jiang, B. Sol-Gel Preparation of PDMS/Silica Hybrid Antireflective Coatings with Controlled Thickness and Durable Antireflective Performance. J. Phys. Chem. C 2010, 114, 19979-19983.

(26) Lippmaa, E. T.; Alla, M. A.; Pehk, T. J.; Engelhardt, G. Solidstate high resolution NMR spectroscopy of spin $1 / 2$ nuclei (13C, 29Si, 119 Sn) in organic compounds. J. Am. Chem. Soc. 1978, 100, 19291931.

(27) Liu, C. C.; Maciel, G. E. The Fumed Silica Surface: A Study by NMR. J. Am. Chem. Soc. 1996, 118, 5103-5119.

(28) Litvinov, V. M.; Barthel, H.; Weis, J. Structure of a PDMS Layer Grafted onto a Silica Surface Studied by Means of DSC and Solid-State NMR. Macromolecules 2002, 35, 4356-4364.

(29) Dong, H.; Lee, M.; Thomas, R. D.; Zhang, Z.; Reidy, R. F.; Mueller, D. W. Methyltrimethoxysilane Sol-Gel Polymerization in Acidic Ethanol Solutions Studied by 29Si NMR Spectroscopy. J. SolGel Sci. Technol. 2003, 28, 5-14.

(30) McNaught, A. D.; Wilkinson, A. IUPAC Compendium of Chemical Terminology (the "Gold Book"), 2nd revised ed.; WileyBlackwell, 1997.

(31) Marmur, A. Underwater superhydrophobicity: Theoretical feasibility. Langmuir 2006, 22, 1400-1402.

(32) Lu, Y.; Sathasivam, S.; Song, J.; Crick, C. R.; Carmalt, C. J.; Parkin, I. P. Robust self-cleaning surfaces that function when exposed to either air or oil. Science 2015, 347, 1132-1135.

(33) Stöber, W.; Fink, A.; Bohn, E. Controlled growth of monodisperse silica spheres in the micron size range. J. Colloid Interface Sci. 1968, 26, 62-69.

(34) Díaz, U.; García, T.; Velty, A.; Corma, A. Hybrid organicinorganic catalytic porous materials synthesized at neutral $\mathrm{pH}$ in absence of structural directing agents. J. Mater. Chem. 2009, 19, 59705979. 